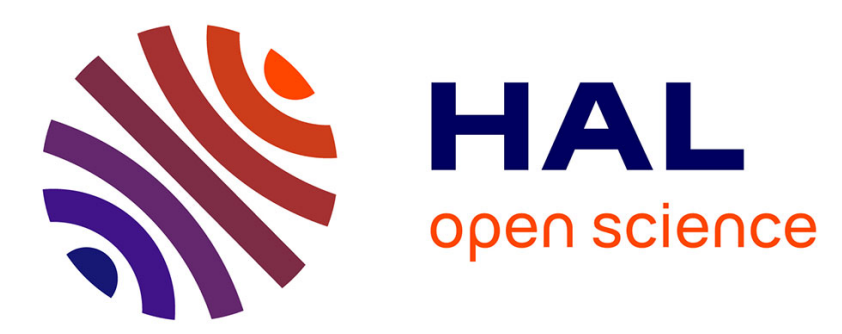

\title{
Bientôt on comptera des dizaines de millions de paysans sans terre en Afrique subsaharienne
}

Christian Bouquet

\section{To cite this version:}

Christian Bouquet. Bientôt on comptera des dizaines de millions de paysans sans terre en Afrique subsaharienne. Hérodote - Revue de géographie et de géopolitique, 2015, 156, pp.93-107. 10.3917/her.156.0093 . halshs-02285458

\section{HAL Id: halshs-02285458 \\ https://shs.hal.science/halshs-02285458}

Submitted on 12 Sep 2019

HAL is a multi-disciplinary open access archive for the deposit and dissemination of scientific research documents, whether they are published or not. The documents may come from teaching and research institutions in France or abroad, or from public or private research centers.
L'archive ouverte pluridisciplinaire HAL, est destinée au dépôt et à la diffusion de documents scientifiques de niveau recherche, publiés ou non, émanant des établissements d'enseignement et de recherche français ou étrangers, des laboratoires publics ou privés. 


\section{Bientôt on comptera des dizaines de millions de paysans sans terre en Afrique subsaharienne}

\section{Christian Bouquet}

Professeur émérite de géographie politique à l'université Bordeaux-Montaigne et chercheur au laboratoire LAM «Les Afriques dans le Monde » (Sciences Po Bordeaux).

On a longtemps conservé l'idée que les paysanneries africaines disposaient d'une telle disponibilité en terres arables qu'elles pouvaient sans difficulté pratiquer l'agriculture itinérante et qu'il n'était pas nécessaire de remettre en cause leur gestion collective des champs. Il n'y avait pas de tensions à propos des limites des parcelles, puisque la cartographie des «terroirs » admettait de larges zones de discontinuité (vastes espaces considérés comme inoccupés), et nombre de chercheurs en étaient restés à la belle utopie selon laquelle, en Afrique, la terre appartient à celui qui la cultive (et les arbres à ceux qui les ont plantés).

Cette perception semble s'être auto-figée en raison de la prise de conscience

tardive - voire retardée par des considérations idéologiques ${ }^{1}$ - que l'Afrique n'était plus souspeuplée et que le rapport entre les surfaces cultivables et leur exploitation n'était plus aussi favorable qu'autrefois. C'est ainsi que le continent, dont la superficie n'avait évidemment pas bougé, était passé de 230 millions d'habitants en 1950 à un milliard en 2010.

À l'évidence, cette mutation profonde devait avoir une incidence directe sur la relation entre l'homme et la terre, et notamment sur la manière dont les sociétés allaient devoir introduire dans leur gestion de l'espace un élément qui leur était peu familier : la limite territoriale. Dès lors, le contrôle collectif des champs cultivables ne pouvait que buter sur la pression des individus, devenus trop nombreux pour une ressource en voie de raréfaction.

S'il est vrai que, selon l'adage populaire, «nécessité fait loi », il a néanmoins fallu précisément écrire ces lois nouvelles, et c'est là que la mondialisation

- définie comme le recouvrement du monde par l'économie de marché - a orienté de manière déterminante l'évolution des campagnes africaines. En introduisant le concept de « sécurisation des terres », c'est-à-dire en imposant le modèle de la propriété privée individuelle, la Banque

\footnotetext{
1 En raison du tabou qui pèse sur le malthusianisme.
} 
mondiale a instillé ${ }^{2}$ au tournant des années 1980 le germe d'une révolution sociale dont on commence à peine à saisir la gravité. Et pourtant les signaux négatifs ont été nombreux, aussi bien autour de la question de savoir « à qui appartient réellement la terre », avec à la clé - par exemple - une guerre civile larvée en Côte d'Ivoire dès les années $1990^{3}$, qu'autour des bénéficiaires potentiels de ces réformes agraires, avec des opérations d'accaparement (land grabbing) par les grands groupes agro-industriels ayant conduit - par exemple - à la chute du président Marc Ravalomanana à Madagascar en 2009. Peut-être convient-il alors de revenir sur ces deux sujets, afin de faire reposer notre hypothèse prospective sur un état des lieux solidement étayé.

\section{Les droits fonciers coutumiers pouvaient-ils résister à la globalisation ?}

On ne sait plus très bien qui a inventé l'expression «sécurisation des terres », mais on a bien compris qu'elle convenait parfaitement au dessein des institutions de Bretton Woods visant à transférer en douceur le modèle de la propriété privée individuelle de la terre sur un continent qui ignorait ce concept. Deux facteurs complémentaires ont pesé dans l'adhésion quasi générale des décideurs à cet aggiornamento majeur: l'explosion démographique et la nécessité d'augmenter sensiblement la productivité des exploitations agricoles, ne serait-ce que pour lutter contre l'insécurité alimentaire.

Pour autant, les chercheurs n'auraient pas dû être pris de court ${ }^{4}$, d'une part, parce que le rouleau compresseur de la globalisation ne pouvait pas laisser subsister des droits collectifs encore très prédominants dans un monde reposant sur le primat de l'individu (a fortiori après la chute du communisme) et, d'autre part, parce que des travaux anciens avaient dès les années 1960 appelé l'attention sur l'inéluctable mise en extinction des droits fonciers coutumiers en Afrique subsaharienne ${ }^{5}$. Car c'est bien le collectivisme agraire qui a prévalu pendant des siècles dans

2 Le président de la Banque mondiale déclarait en 1991 : «Il faut renforcer la protection du régime foncier pour mieux assurer la propriété. (...) Il existe de nombreuses indications que l'agriculteur africain, comme partout ailleurs, répondra à ces mesures d'encouragement » [McNamara, 1991, p. 46].

3 Après la multiplication d'incidents intercommunautaires autour des conflits fonciers, la Côte d'Ivoire a connu un coup d'État militaire en 1999, puis une rébellion en 2002, qui a provoqué la scission du pays pendant cinq ans.

4 Dans un Café-Géo tenu le 15/12/2009 [Charvet et Moreau, 2009], Michel Sivignon souligne que personne ne parlait du land grabbing avant que la presse ne fasse émerger le sujet [Bouquet, 2008-2009].

5 Kouassigan [1966]. La thèse soutenue en 1962 à la faculté de droit de Toulouse par Guy- Adjété Kouassigan contenait à la fois une synthèse très complète des pratiques foncières en Afrique de l'Ouest et une identification précise des facteurs qui allaient conduire à la mutation des droits coutumiers. Il avait également 
cette partie du continent africain. «Il y a un fait qu'il faut toujours avoir à l'esprit pour comprendre les droits fonciers négro-africains, c'est que la notion de propriété individuelle de la terre n'y existe pas. La terre appartient à une communauté (le canton, le village, ou la famille élargie) mais jamais à un individu à titre privatif et exclusif. Tous les membres de la communauté villageoise y ont des droits strictement égaux » [Kouassigan, 1966, p. 54]. Les exemples sont innombrables, que ce soit au Nigeria [Jones, 1949, p. 313] ou en Côte d'Ivoire [Clozel et Villamur, 1902 ; Tauxier, 1932].

Cependant, pour que cette forme de collectivisme agraire ait pu traverser les siècles sans jamais être remise en cause - et avant que la pression démographique impose des infléchissements -, il a fallu qu'elle bénéficie à la fois du poids de l'irrationnel des religions traditionnelles et de la solidité des organisations sociales autour des chefs de terre.

C'est ainsi que les rituels d'occupation de l'espace, observés il y a quelques décennies aussi bien en Afrique de l'Ouest (Côte d'Ivoire) qu'en Afrique centrale (Cameroun), donnaient un caractère sacré et donc inaliénable aussi bien à l'ensemble du terroir contrôlé par la communauté qu'aux parcelles concédées aux familles à l'intérieur de l'ensemble. Chez les Ebrié de Côte d'Ivoire, Okou-Henry Legré fait remarquer : «On demande aux anciens du groupe d'offrir leurs terminaux (ongles, poils du sexe) qu'on enterre avec une plante aux feuilles grasses » [Legré, 2003, p. 47], pratique qui s'apparente aux rituels des Romains (sacrifices humains) ou des sociétés rurales du XI $\mathrm{e}^{\mathrm{e}}$ siècle qui se déplaçaient avec les ossements des ancêtres pour marquer leurs nouveaux territoires.

Si les croyances ancestrales demeurent très prégnantes ${ }^{6}$, malgré les progrès de l'alphabétisation qui est censée faire reculer l'obscurantisme, le rôle des chefferies coutumières n'a cessé d'être battu en brèche, d'abord par le pouvoir colonial puis par les politiques (souvent exogènes) conduites depuis les indépendances. Progressivement, les cadres juridiques ont dû évoluer, notamment parce que les droits coutumiers étaient parfaitement adaptés à une économie rurale fermée d'autosubsistance, mais beaucoup moins à une économie de plantation ouverte sur l'exportation et destinée à un enrichissement individuel ${ }^{7}$. L'esprit communautaire s'est également amenuisé parce que le groupe des origines est devenu quantitativement ingérable et

dépouillé de nombreuses sources anglophones. Cinquante ans après, ce travail mérite d'être attentivement relu.

6 À Madagascar, par exemple, les opérations de cadastrage sont singulièrement compliquées par l'existence de tombeaux familiaux, liés à un culte des ancêtres qui sacralise la terre où ils sont inhumés.

7 Il y aurait lieu de s'interroger sur l'apparente évidence de cette formule, mais il est vrai que la notion d'enrichissement collectif n'a guère connu d'illustrations probantes. 
s'est fractionné en cercles de parenté de plus en plus étroits, mais aussi de plus en plus attachés aux patrimoines individuels. Or les règles juridiques doivent suivre les évolutions de l'ordre social, «sinon le droit finira par être un élément de régression » [Kouassigan, 1966, p. 179]. À la fin des années 1980, le temps semblait donc venu de faire passer la terre des res extra commercium aux res in commercio.

Le processus s'est néanmoins révélé plus compliqué qu'il n'y paraissait. L'inaliénabilité de la propriété collective n'avait d'ailleurs jamais été ébranlée par le pouvoir colonial, qui avait maladroitement tenté d'isoler des «terres vacantes et sans maître » pour en faire des biens domaniaux, mais sans grand succès car s'il peut effectivement exister des terres apparemment vacantes $^{8}$, elles ne sont jamais «sans maître ». On peut néanmoins distinguer «les produits de la culture de la terre qui les porte » [Kouassigan, 1966, p. 210], parce que « toutes les coutumes s'accordent sur ces points. Elles spécifient par exemple qu'une récolte sur pied ne saurait appartenir à celui qui a prêté ou loué le terrain à un exploitant. Le possesseur du droit du sol ne pourra user de celui-ci qu'après enlèvement de la récolte »[Labouret, 1941]. Cette interprétation a ouvert une brèche dans le collectivisme agraire précolonial et colonial, mais quid des plantations, donc des arbustes et des arbres dont la durée de vie peut excéder une génération?

Même si, pour paraphraser François Perroux, la propriété privée est psychologiquement un état d'esprit, la situation du monde paysan africain dans les années 1980 a incité les décideurs de la gouvernance économique mondiale à prendre des précautions avant de faire basculer le continent dans un système de gestion foncière diamétralement opposé à ses valeurs ancestrales : la privatisation de la terre.

\section{Prudence et hésitations des bailleurs de fonds avant le passage à l'acte}

Il s'est probablement trouvé, dans les arcanes des institutions de Bretton Woods, quelques esprits perspicaces pour percevoir les risques d'embrasement social qui allaient apparaître lors de la mise en place des nouvelles lois foncières. C'est pourquoi, à l'initiative éclairée de la Coopération française, et avec l'appui opportun (ou opportuniste) de la Banque mondiale,

8 Les « terres vacantes » sont généralement des jachères utilisées dans le cadre des rotations culturales. Il arrive malheureusement que les organisations internationales les comptabilisent encore aujourd'hui comme des terres cultivables non encore utilisées. 
plusieurs programmes de recherche-action dénommés Plans fonciers ruraux (PFR) ont été lancés dans les années 1980 dans cinq pays d'Afrique de l'Ouest (Bénin, Burkina Faso, Côte d'Ivoire, Guinée et Mauritanie) afin d'établir un état des lieux des droits coutumiers, des modes de délimitation des parcelles, de l'origine des conflits et des processus traditionnels de règlement de ceux-ci. Ont été associés à ces travaux de nombreux chercheurs relevant de l'Institut de recherches pour le développement (IRD), du Groupe de recherches et d'échanges technologiques (GRED) et de l'International Institute for Environnement and Development (IIED), ce qui a renforcé l'approche anthropologique du sujet, en même temps que la bonne conscience des bailleurs de fonds.

Démarrés à la fin des années 1980, ces PFR ont porté sur près d'un million d'hectares, dont 670000 en Côte d'Ivoire. Dans ce pays, où les tensions étaient les plus fortes notamment en raison d'un repli identitaire visant à écarter les allochtones des terres qu'ils avaient pourtant mises en valeur ${ }^{9}$, les travaux du Plan foncier rural avaient ciblé plusieurs zones témoins et touché plus de 300 villages. Malheureusement, le pouvoir de l'époque (Henri Konan Bédié) souhaita aller plus vite en besogne et adopta dès 1998 une nouvelle loi foncière avant que les conclusions n'aient été tirées.

«En Côte d'Ivoire, le Plan foncier rural présentait l'avantage de commencer par un état des lieux, décrivant notamment la manière dont les parcelles pouvaient être cartographiées (pour un cadastrage ultérieur), la nature des droits fonciers traditionnels tels qu'ils étaient appliqués, et les caractères des litiges recensés.

La phase pilote de l'opération (octobre 1989-juin 1996), conduite sur 476000 hectares autour de Korhogo, Bouaké, Abengourou, Daloa et Soubré, avait touché 270 villages, soit environ 27000 personnes. Elle avait amorcé la création de comités villageois de gestion foncière (CVGF) appelés à jouer, plus tard, un rôle important de médiation.

Une phase de consolidation (juillet 1996-juin 1997), financée par l'AFD, avait concerné 157000 hectares nouveaux pour 65 villages autour de Bangolo, Bondoukou, Daoukro et Odienné.

Mais la dernière phase, dite «phase d'extension » (juillet 1997-juin 2002), échoua en raison, d'une part, de la tension sociale qui prévalait autour de l'ivoirité, et donc du concept

9 La multiplication des conflits fonciers, parfois meurtriers, entre des communautés d'origines différentes a fait monter la xénophobie dans le pays. Le repli sur l'«ivoirité » a failli conduire à une guerre civile entre 1996 et 2010 . 
d'autochtonie dans les campagnes, et, d'autre part, du fait que la loi foncière, que le PFR était censé préparer, avait été adoptée par l'Assemblée nationale le 23 décembre 1998. »C. Bouquet [2011, p. 32].

En fait, les PFR n'ont probablement été que des barouds d'honneur pour ceux qui espéraient encore pouvoir trouver un heureux compromis entre l'attachement des paysans à leurs droits coutumiers et les impératifs de l'économie de marché. D'ailleurs, comme une déferlante que rien ne peut arrêter, de nouvelles dispositions législatives contraignantes ont été rapidement adoptées dans les pays de la sous-région : dès 1996 au Burkina Faso, en 2004 au Sénégal, en 2006 au Mali, et en 2007 au Bénin. Dans le reste du continent, la «sécurisation des terres » a été actée de la même manière en 1999 en Tanzanie (Land Village Act), en 2004 en Angola, et en 2005 à Madagascar.

Comme il fallait s'y attendre, l'application concrète de cette nouvelle réglementation a buté sur de nombreuses difficultés, ne serait-ce que sur le simple problème de la délimitation des parcelles. Dans les régions sahéliennes où cultivateurs sédentaires et éleveurs semi-nomades se sont longtemps partagé les mêmes espaces (les premiers pour leurs cultures de saison des pluies, les seconds pour les pâturages avant et après les cultures), à qui allait-on attribuer les nouveaux titres de propriété ?

Par ailleurs, cadastrer des millions d'hectares dans des pays où la notion de limite linéaire n'a guère de sens reste le principal point technique de blocage, notamment à cause des questions fondamentales telles que «qui va le faire ?» (il n'existe pas de topographes en nombre suffisant) et «qui va payer?» (les États n'ayant pas les moyens de faire face à une telle dépense, ce sont les détenteurs des droits qui devront rémunérer les techniciens).

Enfin - et ce n'était pas le moindre obstacle - il a fallu trancher entre le droit du sol (jus soli) et le droit du sang (jus sanguinis). Ce dilemme était particulièrement sensible dans les pays où les fronts pionniers avaient été défrichés par des allochtones, voire des allogènes, pour y développer des cultures de plantation ${ }^{10}$, introduisant par là même une ambiguïté dans l'application des droits coutumiers.

Aussi l'attribution des nouveaux titres fonciers progresse-t-elle très lentement dans les pays où le processus a démarré (Madagascar), et semble au point mort dans les pays où le sujet reste

10 Le cas de la Côte d'Ivoire est emblématique de cette situation. Plusieurs centaines de milliers de planteurs, ivoiriens et étrangers, sont installés parfois depuis deux ou trois générations sur des terres n'ayant pas « appartenu » à leurs ancêtres. 
politiquement très sensible comme la Côte $\mathrm{d}^{\prime}$ Ivoire ${ }^{11}$. Mais si l'application généralisée de ces lois prend du retard, on a rapidement remarqué qu'elles visaient plutôt à ouvrir le marché des terres arables aux grands groupes agro-industriels internationaux qu'à la petite exploitation familiale nationale. C'est ainsi que Pierre-Claver Kobo [2003, p. 29] écrit à propos de la Côte d'Ivoire : «Il ressort clairement qu'au travers de cette loi l'État ivoirien reprend à son compte le projet du colonisateur: asseoir la propriété privée moderne, substituée à la propriété collective coutumière, appréhendée comme un obstacle au développement. »

Autrement dit, le temps des cultures obligatoires imposées aux paysans d'Afrique subsaharienne à l'époque de la colonisation est révolu. On va désormais faire la même chose, mais dans un cadre parfaitement légal, et au nom du « développement ».

\section{La tendance à l'accaparement s'accélère et finira par prévaloir}

Paradoxalement, alors que la proportion de terres immatriculées ne dépassait pas $2 \%$ au tournant des années 2000 , les transactions ont commencé à se multiplier et, peu à peu, à attirer l'attention à cause des superficies sur lesquelles elles portaient.

En effet, en se remémorant l'histoire des campagnes anglaises au $\mathrm{XvI}^{\mathrm{e}}$ siècle et le mouvement des enclosures, les partisans de Rostow et de son «parcours obligé » (1963) imaginaient sans doute déjà qu'on allait assister à une redistribution locale des parcelles, dès que chaque exploitant disposerait d'un titre de propriété : les plus audacieux et les plus nantis rachèteraient les terres aux plus démunis pour se constituer des fermes de dimension rentable. Sachant que les exploitations familiales en Afrique subsaharienne dépassent rarement un hectare, on pouvait prévoir une évolution semblable à celle des campagnes européennes au $\mathrm{XIX}^{\mathrm{e}}$ siècle avec l'émergence de «moyennes propriétés » de l'ordre de 20 à 50 hectares. Notons au passage parce que cela n'a guère été anticipé pour l'Afrique - que ce processus avait inévitablement généré un fort exode rural, absorbé par la révolution industrielle dans les pays du Nord. Et ce segment du «parcours obligé » est encore manquant sur le continent africain.

Or, à peine ouvert par la «sécurisation des terres », le marché du foncier rural africain a essentiellement attiré de très gros investisseurs étrangers et le phénomène de l'accaparement (land grabbing) a interpellé les opinions publiques, au point d'ailleurs de susciter des

11 La loi de 1998 accordait un délai de dix ans à ceux qui souhaitaient faire valoir leurs droits sur « leur » terre. Ce délai a été renouvelé pour dix nouvelles années en 2013. Il semble que nul ne souhaite vraiment avancer sur ce sujet en raison du risque de voir les campagnes s'embraser. 
évaluations chiffrées parfois fantaisistes. Si l'on s'en tient aux données rassemblées par le Comité catholique contre la faim et pour le développement (CCFD), 134 millions d'hectares auraient fait l'objet de transactions en Afrique ${ }^{12}$ entre 2000 et 2010. Compte tenu des réserves généralement exprimées sur les estimations chiffrées, cela représenterait entre 5 et $10 \%$ des terres arables de ce continent ${ }^{13}$.

S'il est vrai que le tableau quantitatif n'est pas consolidé, les principes qui président à de telles opérations sont clairs. Il suffit de citer l'exemple de l'homme d'affaires français Charles Beigbeder qui a commencé en 2008 à investir dans les riches terres cultivables d'Ukraine. Aujourd'hui, après la fusion de sa société Agro Génération avec la firme américaine Harmelia, il contrôle 120000 hectares sur lesquels il produit des céréales à des prix compétitifs - même si les rendements sont moins élevés qu'en Beauce - puisque la terre lui a été concédée à bas prix, que la main-d'œuvre n'y est pas chère, et que la fiscalité y est très avantageuse. C'est le cas d'école par excellence de l'économie de marché élargie à la terre.

Ce qui était possible en Ukraine est désormais encore plus simple à réaliser en Afrique. Sans revenir en détail sur les principales opérations d'accaparement qui ont marqué l'actualité, on rappellera toutefois l'emblématique exemple malgache, où le groupe Daewoo avait entamé en 2008 des négociations directes avec l'État pour cultiver sur 1300000 hectares du palmier à huile et du maïs, c'est-à-dire des produits destinés au marché coréen. Les principaux problèmes posés par le processus d'accaparement s'inscrivaient déjà en filigrane de ce projet de contrat : on allait mobiliser les terres malgaches pour nourrir des populations étrangères («Peut-on nourrir les riches avec les terres des pauvres ? » [Charvet et Moreau, 2009]) et, en introduisant dans l'agriculture des méthodes intensives, on se préparait à chasser $95 \%$ des paysans de leurs anciennes terres $^{14}$ («Doit-on et peut-on éviter la grande évacuation des campagnes africaines et asiatiques ? » [Rouillé d'Orfeuil, 2007]).

Finalement, le projet Daewoo n'a pas abouti, sinon à la chute en mars 2009 du président malgache Marc Ravalomanana qui avait imprudemment engagé le processus. Un groupe indien

12 http://ccfd-terresolidaire.org/IMG/pdf/acc_terres_ccfdjuin2012.pdf et http://landmatrix. org/en/

13 On ne peut manquer de noter le gap relativement important entre ces chiffres et celui des terres immatriculées (2\%). Il s'explique, d'une part, par le fait que toutes les transactions entreprises n'ont pas abouti et, d'autre part, par les « libertés » que se sont données certains États pour concéder des terres sans tenir compte de ceux qui les occupaient.

14 On rappelle qu'en Argentine 4 hommes suffisent pour exploiter 1000 hectares de soja. Sur cette base de calcul, et sachant que l'exploitation familiale moyenne à Madagascar ne dépasse pas 1 hectare, c'est 125000 chefs de famille qui seraient devenus inutiles sur leurs anciennes terres, si le projet Daewoo avait vu le jour. Où seraient-ils allés ? Pour quoi faire? 
(Varun Industry) est arrivé peu après pour produire du riz sur 500000 hectares dans le nord-est de la Grande Île [Bouquet, 2012, p. 395], mais les nouvelles autorités ont préféré être prudentes pendant la période de transition (2009-2013) et rien n'a encore été signé.

On est donc encore probablement loin des 134 millions d'hectares affichés comme potentiellement «accaparés » par des étrangers en Afrique [Arezki, Deininger et Selod, 2011]. Il n'empêche que le mouvement est lancé et sera puissant. En effet, il intéresse au premier chef tous les pays qui manquent de terres arables pour faire face à leurs simples besoins alimentaires. Et si le petit État de Djibouti ne loue que quelques milliers d'hectares en Éthiopie pour compléter son ravitaillement, on imagine les superficies nécessaires pour couvrir la demande de pays comme les États du Golfe, l'Arabie saoudite et d'Asie du Sud-Est. On oublie aussi trop souvent que la Chine doit nourrir $25 \%$ de la population mondiale avec seulement $7 \%$ des terres arables de la planète. Elle figure en bonne place sur les tableaux du land grabbing ${ }^{15}$.

D'autant plus que les besoins alimentaires ne sont pas les seuls moteurs de ces transactions, et les États n'en sont pas les seuls acteurs. On trouve aussi les fonds d'investissement et les multinationales de l'agrobusiness, qui se livrent tout simplement à de gigantesques spéculations, notamment dans le secteur des agrocarburants. Les terres africaines propices au maïs, à la canne à sucre et/ou au palmier à huile sont particulièrement recherchées, en particulier celles qui sont relativement riches et bien alimentées en eau. Les 100000 hectares du projet libyen Malibya sont soigneusement circonscrits dans le Macina malien ${ }^{16}$, c'est- à-dire le delta intérieur du Niger où les débordements saisonniers assurent une fertilité optimale en pleine zone sahélienne. Certes, la chute de Kadhafi a retardé le déploiement complet du programme, mais il a bel et bien démarré ${ }^{17}$ et il réactive le souvenir du temps où les grands nomades du désert venaient se servir en blé dur dans les oasis où travaillaient leurs esclaves du Bîlâd as Sûdân.

À l'appui de tous ces projets, la plupart des économistes et des bailleurs de fonds avancent l'argument parfaitement recevable selon lequel il est temps de rendre davantage productive l'agriculture africaine, fût-ce pour l'exportation. La «sécurisation des terres » est donc un

15 En prenant toutes les précautions nécessaires, on pourra se reporter utilement aux tableaux chiffrés et aux cartes proposés par les sites http://www.grain.org et http://www.farm- landgrab.org

16 On trouvera de bonnes cartes dans un article de Florence Brondeau [2010]. http://echogeo.revues.org/12008

17 http://farmlandgrab.org/post/view/22724-office-du-niger-la-prospection-d-opportunites- nouvelles On note que ce sont des Chinois qui opèrent (pour le compte des Libyens ?) sur les 25000 hectares réellement concernés, et qu'ils visent plus particulièrement la canne à sucre. 
impératif absolu que plus personne ne semble remettre en cause. Le modèle unique aurait-il atteint un point de non-retour?

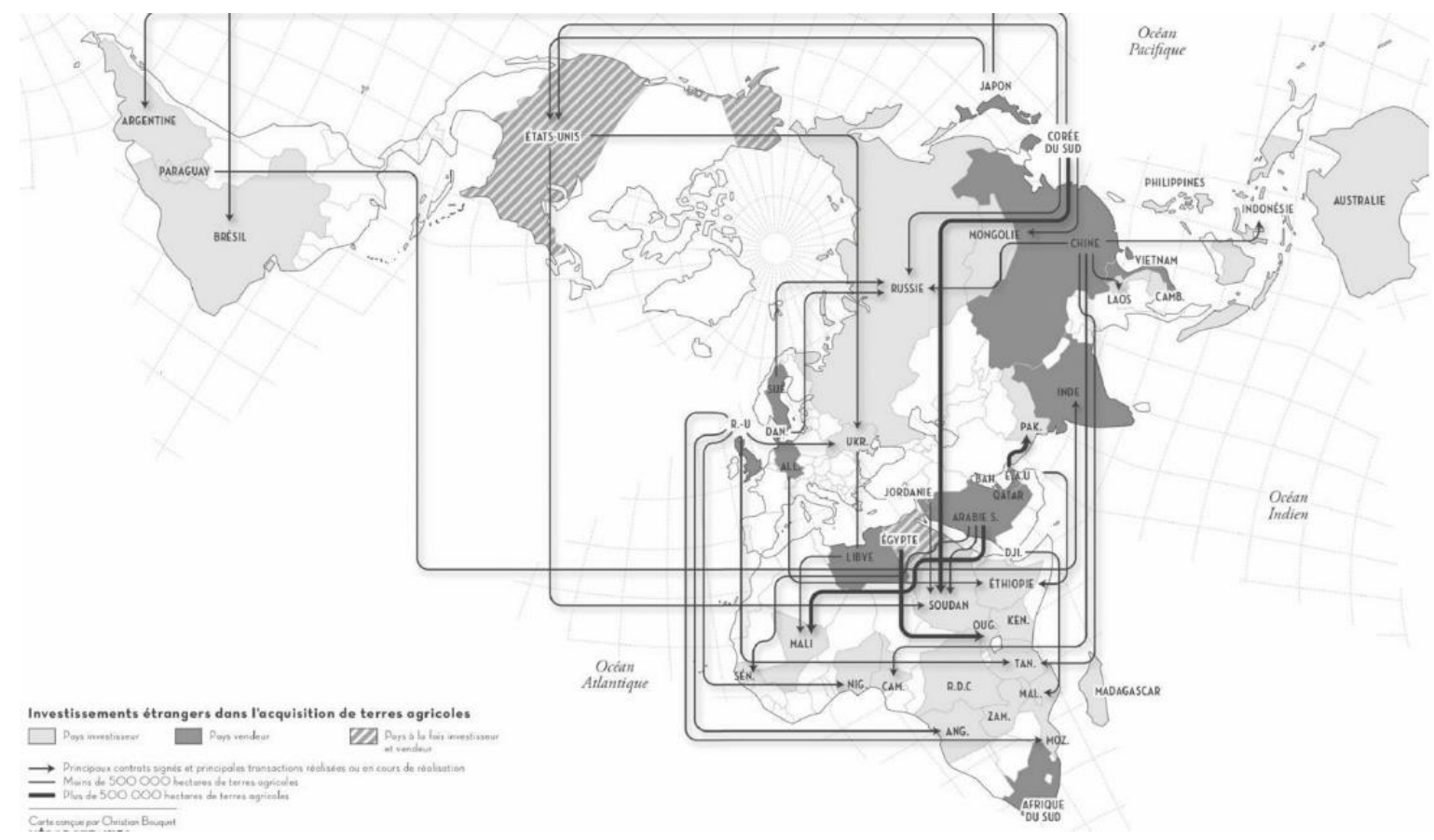

\section{Tardive prise de conscience des risques, timidité des solutions alternatives}

En s'introduisant progressivement dans le paysage économique et sociopolitique africain, le mécanisme de « sécurisation des terres » a surtout secoué les bases du droit foncier coutumier, et c'est dans ce registre que les résistances ont d'abord été les plus fortes. Il est vrai que le passage sans trop de transition du collectivisme agraire à la propriété individuelle pouvait paraître brutal. Or, parallèlement à ce bouleversement, les conséquences les plus graves du processus ne semblent pas avoir été immédiatement perçues. Il était pourtant clair que la distribution de titres fonciers aux petits paysans allait générer une concentration des terres excluant rapidement la plupart d'entre eux du monde rural.

Les manifestations de colère ont donc été tardives, et les premières d'entre elles ont été ignorées des médias ${ }^{18}$. Mais les progrès, même relatifs, de la démocratie et de la liberté d'expression dans un grand nombre de pays africains ont fini par ouvrir la voie à des petits mouvements de résistance, dans lesquels s'est invitée Via Campesina. La puissante confédération est très 
présente dans les rencontres militantes africaines comme lors de l'appel de Dakar « contre les accaparements de terre » en février 2011, ou pendant la Conférence paysanne internationale qui s'est tenue en avril 2012 au Mali. Elle est également active dans les manifestations - encore clairsemées - de paysans spoliés au Mali, à Madagascar, au Sénégal ou en Côte d'Ivoire ${ }^{19}$. Créée en 1993 au Nicaragua, Via Campesina défend la sécurité alimentaire mais aussi les petites et moyennes exploitations paysannes face aux géants de l'agrobusiness et elle est assimilée, à tort ou à raison, à la lutte des paysans sans terre. Son apparition sur les théâtres africains est donc hautement symbolique de la prise de conscience progressive des enjeux.

Certes, comparativement à l'Amérique latine, la fermentation sociale des paysans sans terre n'est pas encore très sensible en Afrique, à l'exception des anciens pays d'apartheid (Zimbabwe notamment). Pourtant, c'est une bombe à retardement dont le tic-tac commence à peine à être compris dans sa véritable dimension, c'est-à-dire l'exode massif de dizaines, voire de centaines de millions de paysans privés (légalement) de leurs terres par le processus de concentration qui est inscrit entre les lignes de la globalisation. Et, au bout de cet exode, les métropoles africaines n'auront rien à leur proposer d'autre que de s'entasser dans des bidonvilles surchargés, dans un continent qui se distingue déjà par un nombre record de populations déplacées ou réfugiées.

Certaines organisations internationales, y compris institutionnelles, commencent à tirer la sonnette d'alarme. La FAO en appelle à une stratégie «gagnant-gagnant » qui suppose des «contrats équilibrés ${ }^{20} »$. Quelques ONG engagées ont plaidé pour l'adoption par la FAO (Rome, 2012) de «directives volontaires ${ }^{21}$ » visant à réguler la spéculation et à imposer des règles aux multinationales. En pointe sur le sujet, le CCFD demande clairement le changement de modèle économique et la mise en place d'une agriculture paysanne basée sur les petites et moyennes exploitations familiales.

Le point de non-retour serait-il déjà atteint? Peut-être pas, dans la mesure où certains économistes commencent à introduire dans leurs calculs de rentabilité le fameux «filet de sécurité » qu'il faudra obligatoirement mettre en place un jour pour accompagner ces dizaines de millions de familles amenées à quitter leurs terres sans savoir où aller ni quoi faire. Le coût de la prise en charge de ces déplacés devrait être intégré au prix de revient de la tonne d'huile de palme, de maïs ou de tout autre produit issu des terres accaparées. Les opérations de land

19 http://www.paixetdeveloppement.net/afrique-foncier-etats-et-populations-problemes- fonciers-gestiondes-terres-conflits-et-perspectives/

20 http://www.fao.org/news/story/fr/item/74396/icode/

$21 \mathrm{http} / / /$ www.fao.org/docrep/016/i2801f/i2801f.pdf 
grabbing apparaitront sans doute alors comme nettement moins rentables. Mais cette proposition, qui s'apparente au principe de l'eau virtuelle ${ }^{22}$, frise l'utopie.

Elle suppose d'ailleurs, en aval, que cette prise en charge des paysans sans terre consiste en emplois réels et ne demeure pas du simple assistanat.

\section{Conclusion}

Parmi les modèles que les pays du Nord ont transférés dans les Suds sans trop de précautions l'État, la nation, la démocratie, la décentralisation... -, la propriété privée de la terre est peutêtre le plus destructeur en termes de valeurs traditionnelles, parce que le collectivisme agraire était profondément enraciné dans le temps et dans l'espace en Afrique. Mais la très forte croissance démographique, marquée par le quadruplement de la population en moins de soixante ans, a rendu les communautés paysannes plus sensibles à l'occupation de l'espace. Elles ont eu l'obligation de tracer des limites aux parcelles et d'intensifier les méthodes pour augmenter les rendements.

En introduisant dans l'économie agraire le titre de propriété pour chaque champ ou parcelle, la Banque mondiale a ouvert la voie à la concentration des terres, selon un mouvement presque simultané d'appropriation/dépossession puisque les plus petits exploitants ne disposent pas de superficies suffisantes pour que leurs efforts de productivité soient rentables. Le seul avantage du mécanisme de « sécurisation des terres » est de leur assurer un petit gain financier lors de la vente. Ensuite, ils seront ruinés, sans terre, et sans perspective d'avenir sur place car les grandes exploitations seront hypermécanisées.

Osons une cartographie prospective : dans vingt ans, peut-être moins, l'écharpe ouest-africaine couvrant l'isohyète $600 \mathrm{~mm}$ sera devenue une immense cotton belt aux mains des multinationales transfuges des États-Unis (où les cotonculteurs ne seront plus subventionnés). Les terres leur auront été concédées à bas prix, la main-d'œuvre sera bon marché et peu nombreuse du fait de la mécanisation, et la fiscalité attrayante. Devenus inutiles sur leurs anciennes terres, les ex-paysans et leurs familles s'entasseront par millions dans les bidonvilles des nouvelles mégapoles côtières.

22 Le concept d'eau virtuelle associe au coût d'un produit le prix de l'eau qu'a nécessité sa production. 


\section{Bibliographie}

ARESKi R., DeININGER K. et SElod H. (2011), «La course aux terres. Quelques éclairages empiriques », Afrique contemporaine, $\mathrm{n}^{\circ} 237$, p. 131-134.

BouQueT C. (2008), « Néocolonialisme agraire », Le Monde, 19 décembre 2008. BouQUET C. (2009a), «L'agriculture délocalisable », Sud-Ouest, 12 avril 2009.

Bouquet C. (2009b), « L’Afrique, dernier Far-West », Jeune Afrique, n 2531,12 juillet.

Bouquet C. (2010), «La bataille pour la terre arable», in BADIE B. et VidAL D., La Fin du monde unique, La Découverte, Paris, p. 211-216.

Bouquet C. (2011), Géopolitique de la Côte d'Ivoire. Le désespoir de Kourouma, Armand Colin, Paris.

Bouquet C. (2012), «En Afrique, les terres appartiendront à ceux qui les achètent », BAGF, 2012-3, p. 388-398.

BRONDEAU F. (2010), « Les investisseurs étrangers à l'assaut des terres agricoles africaines », EchoGéo 14/2010.

Charvet J.-P. et Moreau S. (2009), «Peut-on nourrir les riches avec les terres des pauvres ? », Café-Géo, n 1717,15 décembre 2009.

Clozel J. et Villamur R. (1902), Les Coutumes indigènes de la Côte d'Ivoire, Challamel, Paris.

JONES G. I. (1949), « Ibo land tenure », Africa, $\mathrm{n}^{\circ} 19$.

Ково P.-C. (2003), « La loi ${ }^{\circ}$ 98-750 du 23/12/1998 sur le domaine foncier rural : une lecture critique d'une loi ambiguë », Regards sur le foncier rural en Côte d'Ivoire, CERAP, Abidjan, p. 21-43.

KouASSIGAN G.-A. (1966), L'Homme et la Terre, ORSTOM, Office de la recherche scientifique et technique outre-mer, Marseille.

Labouret H. (1941), Paysans d'Afrique occidentale, Gallimard, Paris.

LEGRÉ O.-H. (2003), « Le rôle de la chefferie traditionnelle dans la gestion foncière en Côte

d'Ivoire », Regards sur le foncier rural en Côte d'Ivoire, CERAP, Abidjan, p. 45-50.

MCNAMARA R. S. (1991), «La crise du développement en Afrique: stagnation de l'agriculture, explosion démographique et détérioration de l'environnement», Actes du Forum de Tokyo sur les stratégies de développement des pays les moins avancés pendant les années 1990, FENU, Fonds d'équipement des Nations unies, p. 29-72.

Rouillé-D’ORfeuil H. (2007), «Doit-on et peut-on éviter la grande évacuation des campagnes africaines et asiatiques ? », Économie rurale, $\mathrm{n}^{\circ} 300$, p. 124-128.

TAUXIER L. (1932), Religion, mours et coutumes des Agni de Côte d'Ivoire, Geuthner, Paris. 